\title{
ESTHER HERNÁNDEZ
}

\section{El Vocabulario náhuatl de Molina frente al Vocabulario de Nebrija ${ }^{1}$}

Parece que fue el Vocabulario en lengua castellana y mexicana el primer diccionario que se imprimió en América. Su primera edición data de 1555 y poco después salió la segunda de 1571 para la que su autor aumentó en gran medida las entradas de la primera edición castellano-mexicana y a la que añadió la parte mexicano-castellana ${ }^{2}$.

El autor, fray Alonso de Molina, viajó a la Nueva España siendo un niño y aprendió en su infancia la lengua de los nahuas. Fue considerado por sus contemporáneos como uno de los españoles que mejor la conocía y este conocimiento de la lengua indígena fue determinante en su vida, puesto que la dedicó a escribir una importante obra lingüístico-evangélica en torno al náhuatl, en la que destacan dos doctrinas, dos confesionarios, un arte y los dos vocabularios.

Su labor estuvo guiada por la voluntad de servir en la comunicación entre los indígenas y los españoles; pero no vamos a tratar aquí los condicionantes socioculturales que facilitaron la aparición de un diccionario que valora y potencia una lengua indígena: han sido ya bien estudiados hechos importantes en este sentido, como el celo misionero de los franciscanos-cuya acción lingüística en el México Central fue muy destacada-; o las circunstancias político-ideológicas-las creencias del humanismo europeo-, que a mediados del XVI hacen viable el estudio y análisis de las lenguas generales en el Nuevo Mundo.

1 Este trabajo está basado en la comunicación que presentamos en el $V$ Congreso Internacional de "El español de América", Universidad de Burgos, 6-10 de noviembre de 1995, titulada "El léxico innovador en el Vocabulario en lengua castellana y mearcana de Molina (México, 1571)", inédita.

2 Fray Alonso de Molina, Vocabvlario en lengva castellana y mexicana, México, 1555 [ejemplar de la Biblioteca Nacional de Madrid, con número de referencia R-8564]; $i$ i., Vocabvlario ..., México, 1571 [ejemplar de la BN, con número R-552]. Para esta última, hemos empleado la edición facsimilar de Porrúa ( $2^{n}$ ed., México, 1977). 
Este trabajo pretende mostrar la originalidad de este diccionario y señalar que es una obra merecedora de un lugar importante en la lexicografía española. Para ello, en primer lugar, vamos a intentar describir cómo Molina copia el Vocabulario de Nebrija y qué edición empleó para ello ${ }^{3}$. En segundo lugar, intentaremos explicar su técnica lexicográfica, esto es, cómo confeccionó las diversas entradas de su diccionario a partir de las de Nebrija. Distinguiremos las entradas nuevas patrimoniales que introduce Molina tras comparar las entradas de las tres primeras letras de ambos diccionarios. Finalmente, estudiaremos parte de este léxico nuevo indagando su historia y comprobaremos si se documenta en la época.

Es bien sabido que la obra lingüística de Nebrija constituyó el punto de partida para reducir a arte gramatical las lenguas americanas y codificar su léxico. Este hecho tiene gran trascendencia en la lingüística americana, hasta el punto de que implicó, en cierta medida, la perduración de muchas lenguas ${ }^{4}$. En el caso concreto de fray Alonso de Molina, su obra lexicográfica se reconoce como la autoridad de la lengua náhuatl clásica, y a ella aún acuden los lexicólogos o etimólogos indoamericanistas ${ }^{5}$.

En otra ocasión nos ocupamos del léxico indígena que Molina incluye como entrada y en el cuerpo de las definiciones de su diccionario ${ }^{6}$. Comprobamos entonces que empleó, con bastante frecuencia, 70 vocablos indígenas diferentes en el texto castellano, prestados de las lenguas antillana y azteca (aguacate, batey, camote); a 17 de ellos ya les reservó una entrada (batata, bohío, cacao), confirmando, pues, de manera clara, su uso en español.

Como hemos señalado antes, nos centramos ahora en el estudio del léxico patrimonial innovador, en concreto el que registra como lema en la primera parte del Vocabulario; innovador en la medida en que discrepa de su modelo, el Vocabulario de romance en latín de Nebrija. Porque, después de comparar

3 Elio Antonio de Nebrija, Vocabulario español-latino, [Salamanca, ¿1495?], Madrid, Real Academia Española, 1951 [nueva impresión, 1989]; id., Vocabulario de romance en latín, trascripción crítica de la edición revisada por el autor (Sevilla, 1516) e introducción de G. J. MacDonald, Madrid, Castalia, 1981.

4 Véanse: Ascensión Hernández de León Portilla, "Nebrija y el inicio de la lingüística mesoamericana" Anuario de Letras, XXXI, 1993, pp. 205-223; José Luis Martínez, "Nebrija en México", Nueva Revista de Filología Hispánica, XII, 1, 1993, pp. 1-17.; José G. Moreno de Alba, "Antonio de Nebrija en España y América", Cuadernos Americanos, 35, 1992, pp. 134-144.

5 Sobre su recepción, véase Manfred Ringmacher, "El Vocabulario náhuatl de Molina leído por Humboldt y Buschmann", en: Klaus Zimmermann (ed.): La descripción de las lenguas amerindias en la época colonial, Vervuert, Iberoamericana, 1997, pp. 75112.

6 Esther Hernández, "Vocabulario en lengua castellana y mexicana" de fray Alonso de Molina. Estudio de los indigenismos léxicos e indice de las voces españolas internas, Madrid, CSIC, 1996. 
las tres primeras letras de los dos diccionarios (A, B y C), confirmamos que lo imita sistemáticamente, y él mismo lo cita en el Prólogo. A pesar de esta deuda, hay algunas diferencias, en cuanto a extensión y ordenación, que otorgan a Molina cierta originalidad. Este hecho permite situarlo como un autor de importancia singular en la lexicografía española, según señala Manuel Alvar:

(...) Fray Alonso era un gramático excepcional, igual que lo fue también como lexicógrafo y su nombre debe figurar entre los más ilustres de nuestra cultura?

Molina, al imitar el diccionario de Nebrija, hace lo que poco después harían los lexicógrafos de otros romances, como C. de Las Casas (1570), C. Oudin (1607), R. Percival (1591), J. Palet (1604): adaptar el Vocabulario de Nebrija a los otros idiomas. Sin duda, en la historia de la lexicografía sucede que los autores se copian unos a otros, quitando y añadiendo a los trabajos de sus antecesores lo que creen adecuado a su época o a los fines que se proponen. Pero Molina no se limita a dar la equivalencia nahua de la entrada castellana de Nebrija, sino que suprime, extiende o añade entradas, seguramente para acercar el léxico a la realidad novohispana de mediados del siglo XVI. Esta aportación es muy interesante para conocer aspectos de la historia del español en América. Además, hemos tenido ocasión de comprobar que Molina fue muy riguroso en su quehacer lexicográfico y demostró un gran conocimiento de la lengua española al adaptar el tesoro léxico a la realidad americana . Por otro lado, sobre la enorme tarea de dar equivalencias aztecas, él mismo manifiesta en el prólogo: "me ha costado el trabajo que nuestro Señor sabe, y los que lo entienden podrán imaginar".

Las ediciones que hemos cotejado son la de Salamanca (¿1495?) y la de Sevilla (1516) del Vocabulario de Nebrija, y las dos de Molina impresas en la ciudad de México, la de 1555 y la de 1571.

Tanto el diccionario de Nebrija como el de Molina alcanzaron un notable éxito en su tiempo. Asimismo, los dos tuvieron ocasión de modificar la extensión de la obra para su segunda edición. Molina añade más de cuatro mil vocablos, además de la parte mexicano-castellana; así, en el prólogo de esta parte, él mismo señala:

(...) acorde de hazer esta segunda impression, mejorando la obra que auia principiado en dos cosas. La via, en que al Vocabulario impresso entel ano de cincuenta y cinco que comiença en romançe, añadi [como parecera] mas de quatro mil vocablos.

7 Manuel Alvar, "Nebrija y tres gramáticas de lenguas americanas (nádıuatl, uncohua y chibcha)", en: M. Alvar (coord.): Estudios nebrisenscs, Madrid, Eds. ('ultura llispinica. 1992, p. 332. 
La otra, en componer e imprimir estotro Vocabulario que comiença en lengua Mexicana.

En cambio, Nebrija añade pocos vocablos en 1516 , mientras que suprime bastantes voces ${ }^{8}$.

Nuestro procedimiento de análisis ha consistido en la comparación de las entradas de 1571 y las de ¿1495?. Distinguimos las que contienen lema nuevio y comprobamos si ya estaban en la edición de Molina de 1555 (véase anexo). Paralelamente, hemos averiguado si estaban las voces que Nebrija había suprimido de la edición de 1495 para redactar la edición de 1516. Como resultado del cotejo, advertimos que Molina utilizó más la edición segunda del Vocabulario español-latino de Nebrija, tanto en 1555 como en 1571, ya que Molina no incluye casi ninguna de las suprimidas. Además, otro hecho viene a apuntar en la misma dirección y hace que Molina se acerque más a la segunda edición, como es el de no dar cabida en su diccionario a los nombres propios y a los derivados de éstos, parte importante de la primera edición nebrisense. Aunque no es descartable que utilizara las dos, parece lógico pensar que Molina entendiera que la segunda edición, revisada por el propio autor, sería la más autorizada.

En el diccionario de Nebrija, como en el de Molina, se distinguen las entradas que introducen un lexema principal de las entradas siguientes, pertenecientes a la misma familia léxica, pero con alguna matización morfológica o semántica. Tales matizaciones, hechas sin duda por la necesidad de ajustar la equivalencia a la segunda lengua-la latina en el caso de Nebrija, la mexicana en el de Molina-, explican que en la mayoría de las entradas haya más de una palabra.

Hay artículos que son idénticos a los del repertorio de Nebrija; otros se inspiran claramente en él, pero están adaptados a las características formales de la lengua azteca; el resto son incorporaciones del lexicógrafo franciscano. En función de su distancia de Nebrija, se puede hacer la siguiente clasificación de las entradas del vocabulario de Molina: a) entradas coincidentes, b) entradas diferentes y c) entradas nuevas. Desde un punto de vista cuantitativo, las más numerosas son las diferentes, seguidas de las coincidentes y las nuevas.

Para ilustrar esta clasificación vemos, en el siquiente cuadro, las entradas de los primeros artículos de la letra $\mathrm{B}$ :

8 Según los trabajos de María Lourdes García Macho, "Novedades léxicas en el Vocabulario español-latino de 1516", Ínsula, 551, 1992, pp. 13-14; id., "Sobre los vocablos suprimidos en la edición de 1516 del Vocabulario español-latino de Nebrija", Anuario de Letras, XXXI, 1993, pp. 133-159. De la misma autora es el El léxico castellano de los Vocabularios de Antonio de Nebrija: Concordancia lematizada, 3 vols., Hildesheim / Zürich / New York, Olms-Weidmann, 1996. 


\begin{tabular}{|c|c|}
\hline Nebrija & Molina \\
\hline Bacia aquello mesmo es que bacin & Bacin de barbero o bacia \\
\hline Bacin para lavar los pies & Bacin de barro o lebrillo \\
\hline Bacin para esto mesmo & Bacin o servidor \\
\hline \multicolumn{2}{|l|}{ Bacin como servidor } \\
\hline \multicolumn{2}{|l|}{ Bacin aqueste mesmo } \\
\hline \multicolumn{2}{|l|}{ Bacinete armadura de cabeça } \\
\hline Baço parte de la assadura & Baço \\
\hline Baço aqueste mesmo & Baço, cosa algo negra \\
\hline \multicolumn{2}{|l|}{ Baço cosa un poco negra } \\
\hline & $\begin{array}{l}\cdots \\
\text { Baçucar }\end{array}$ \\
\hline & Baçucada cosa \\
\hline & Baçucador \\
\hline & Baçucadura \\
\hline Bachiller & \\
\hline Badajo de campana & Badajo de campana \\
\hline
\end{tabular}

Mediante este pequeño grupo de lemas reflejamos los criterios de Molina:

1) no copia todas las entradas de su modelo, como en bachiller

2) añade lexemas distintos, como el verbo baçucar.

3) copia de manera idéntica algunas entradas, como baço, cosa algo negra o badajo de campana.

4) se inspira en los lexemas registrados por Nebrija, pero hace modificaciones diversas, de ampliación o reducción, semántica o morfológica, como en el resto de los lemas. 
Su técnica lexicográfica seguiría, por tanto, los pasos siguientes. En primer lugar, copia entradas del diccionario de Nebrija para las que da la equivalencia en azteca. A partir de ese lexema principal, recoge entradas diferentes, derivadas de las de Nebrija, pero que difieren en aspectos morfológicos o semánticos. Finalmente, en algunos casos, no registra ciertos términos, mientras que en otros incorpora nuevas unidades léxicas no incluidas por Nebrija. Este último grupo es importante porque se podría suponer que los vocablos de los artículos nuevos pertenecen a su variedad idiomática $\mathrm{y}$, en consecuencia, pueden ser característicos del léxico novohispano.

- Las novedades léxicas introducidas por fray Alonso son abundantes (véase anexo) $)^{9}$. En su quehacer como lexicógrafo, Molina decide amoldar el acervo léxico que recibe de Nebrija a su propia realidad lingüística. A continuación, hemos comprobado si las palabras que agrega tienen documentaciones contemporáneas. Con ello, pretendemos saber si en su labor como lingüista-lexicógrafo acertó al reservar entradas para voces que estaban en uso en México.

Los diccionarios consultados han sido el DHist, el DCECH de Corominas y Pascual, materiales inéditos del NTLE, el Tesoro de Covarrubias y el Diccionario de Autoridades. También hemos utilizado el Universal Vocabulario de Alfonso de Palencia y el $L H A$ de Boyd-Bowman del XVI ${ }^{10}$.

Gran parte de las palabras incluidas por Molina que Nebrija no registró tienen testimonios coetáneos. Algunas fueron introducidas en el siglo XV, como el grupo de los cultismos precedidos por ad- latino, cuya primera aparición en un diccionario tiene lugar en el de Molina. Se trata de voces hoy tan comunes como admirar, admitir, adquirir, advertir o adviento. También son neologismos del XV los términos: adular, afable, afeminado, en su acepción de 'cobarde', y agonía ${ }^{11}$.

9 Hay que insistir en que nos ocupamos sólo del estudio contrastivo de las entradas lexicográficas. Sobre el léxico general del diccionario, véase E. Hernández, "Palabras del siglo XVI", Revista de Filología Española, LXXVI, 1-2, 1996, pp. 171-175. Sobre el léxico que se aparta de la norma de la época, véase E. Hernández, "Algunas particularidades léxicas del primer diccionario americano", Miscelánea léxica en memoria de Conchita Serrano, Madrid, CSIC, 1999, pp. 339-344.

10 Joan Corominas y Juan Antonio Pascual, Diccionario crítico etimológico castellano $e$ hispánico, Madrid, Gredos, 1987-1991 [DCECH]; materiales inéditos del proyecto Nuevo Tesoro Lexicográfico Español (1490-1726), dirigido por Lidio Nieto Jiménez y Manuel Alvar Ezquerra [NTLE]; Sebastián de Covarrubias, Tesoro de la lengua castellana o española, [Madrid, 1611], ed. de Martín de Riquer, Barcelona, 1987 [Tesoro]; Real Academia Española, Diccionario de la Lengua Castellana, llamado de Autoridades, ed. facsímil, Madrid, Gredos, 1979 [4ª reimpresión] [Aut.]; John M. Mill (ed.), "Universal Vocabulario" de Alfonso de Palencia. Registro de voces españolas internas, Madrid, RAE, 1957; Peter Boyd-Bowman, Léxico Hispanoamericano del siglo XVI, Londres, Támesis, 1971 [LHA].

11 En las entradas de la letra A, Molina incorpora muchas locuciones del tipo abarrisco, 
Siguiendo con la documentación del DHist, comprobamos que Molina registra otras voces características del Siglo de Oro, como abobamiento, abroquelarse, absorto, abultar, acevilarse 'acivilarse', acial, aconsejar, acortar, ahusar y amohinar. Hay incluso voces cuyos primeros testimonios se han hallado en América; a Molina, de hecho, le corresponde la primera documentación de los términos acelerarse 'apresurarse', acorrucado 'encogido', adiestrar, agaçaparse, adive para 'coyote'. Otros autores de Indias son los primeros en escribir las palabras: aficionarse, aguacero, alijar 'alijar navío' y alquilarse.

Muchas de estas voces siguen vigentes en el español actual y otras lo estaban entonces. En cualquier caso, es Molina la primera autoridad lexicográfica que las documenta. En este sentido, parece evidente que el Vocabulario de Molina es una obra original, por lo que reclamamos para él un lugar destacado en la historia de la lexicografía española.

\section{Anexo}

Incorporaciones léxicas del Vocabulariò de Molina (México, 1555 y 1571) ${ }^{12}$

Abarrisco*; Abiuar a otro*; Abocanadas echar algo*; Abochornarse las miesses o secarse por falta de agua*; Abouamiento o embelesamiento; Abroquelarse. busca escudarse*; Absorto estar en algun negocio; Abstenerse de algo, o yrse ala mano; Abuen tiempo*; Abultar con pomposos ornamentos*; Acelerarse*; Aceuilarse o apocarse*; Aconsejar*; Acorrucado o encogido; Acortar o achicar; Acostamiento o soldada; Acostumbrar o ser común prouerbio y manera de dezir; Acreedor; Acucharrada cosa; Adalid*; Aderechas; Adeseo*; Adiestrar ciego*; Adive; Admirar, escandalizar o espan- tar a otro; Admitir a alguno a alguna cosa*; Adquirir con trabajo lo necessario a la vida*; Aduertir a otro, paraque ande sobreauiso enlo que le conuiene; Aduiento*; Adular. busca lisongear*; Aello, sus compañeros, ea ea a ello.*; Aempuxones*; Aescondidas*; Aessotra parte, o a essotra vanda*; Aesta hora*; A este tiempo, o aeste mismo tiempo*; Afable persona*; Afamarse; Afeminado; Aficionarse o aplicarse a algo; Aforrar algo*; Afrentar a otro*; Agaçaparse*; Agonia; Aguacero*; Aguardar o esperar a otro conla comida o con otra cosa assi; Ahermosear o hermosear algo*; Aho-

abocanadas, aderechas, aescondidas, ahurtadillas, etc. apartándose de Nebrija. Su estudio lo concebimos más bien dentro de la palabra que sigue a la preposición, por lo que ahora las pasamos por alto.

12 Con asterisco las entradas que aparecen en la edición de 1555. Además, sólo consignamos las entradas con lexema nuevo, prescindiendo de las que lo amplían o matizan. 
rrar; Ahurtadillas*; Ahusar algo; Ajena cosa*; Aizquierdas hazer algo; Ala mano derecha; Alançar de si algo; Ala oreja; Alcabo o al fin; Alcontrario; Alderredor; Alijar nauio*; Alos lados*; Alos pies de alguno*; Al principio*; Alquilarse*; Alreues; Altercar; Al tercero dia*; Al tercero año*; Alto, aello, sus aello, ea ea aello*; Altoçano o rebenton; Aman derecha*; Aman yzquierda*; Amedia noche*; Amohinar y moler aotro*; Amojonar; Amortajar muerto*; Anhelar mucho por algo; Antantier*; Antenoche*; Antepecho de açotea*; Apalancar*; Apalear*; Aparecer o manifestarse; Apechugar con otro; Apedaços sacar el niño muerto del vientre; Apezgar o apretar algo con las manos*; Aplacar a alguno; Aplicada cosa o dedicada para alguna persona*; Apocarse o aceuilarse en lo que se haze o dize*; Apostar*; Aprensar*; Apuñalado; Apuntalar algo; Aqual de dos*; Aque hora*; Aque o paraque?*; Arcabuz o escopeta*; Arista; Armeja*; Armiño*; Arreburujar algo, assi como papel, manta, estera o cosa semejante.*; Arregaçarse el varon*; Arrobarse o transportarse*; Arrodelarse; Arroyo; Arrollar manta, estera, papel o cosa semejante*; Asadura de animal; Asaetar; Asaz; Asementarse; Asosegar y quietar a otro; Asqua o brasa*; Asqueroso; Assegurar con engaño a alguno; Assosegarse el ayre y la tempestad*; Atentar con la mano, buscando algo*; Atesar o bruñir algo, o alimpiar mucho alguna cosa; Atesorar*; Atestiguador; Atiempo o coyuntura*; Atroche moche*; Atuertas; Aue- nencia o cosa semejante*; Auentajar o sobrepujar a otro*; Auer debaxo exemplo de hazer penitencia los sanctos pasados*; Aumentar; Auergonçar a otro; Auezindarse o detenerse en algun lugar; Auisar a otro*; Axi o pimienta desta tierra*; Azero; Azial*; Baçucar*; Balsa*; Bannas hazer o diuulgaciones alos que se quieren casar*; Barriga o vientre*; Basta*; Bastarme algo*; Bastante ser para algo*; Batata*; Batea*; Batei, juego de pelota con los quadriles, o el mesmo lugar donde juegan este juego*; Bezerro*; Bizma o bidma*; Blasonar o jactarse de su nobleza y gran linaje*; Bocarriba estar la vasija*; Bocabaxo estar assi*; Bodoque*; Boquituerto*; Bracelete de oro*; Breuaje que hazen de cierta harina de chia*; Bruñir lo encalado*; Buhio o casa de paja*; Buzano que anda debaxo del agua*; Cacao, almendra y moneda*; Cacarear la gallina*; Caducar*; Caldo de carne*; Camellon de tierra*; Cancelar escriptura*; Carienxuto*; Cariredondo*; Caritriste*; Carlear*; Carimenudo*; Caro en precio*; Castañeta*; Castizo*; Cauallete de pared*; Coa o pala para cauar o deseruar*; Cocar la ximia*; Compañon o cojon*; Comprehender lo que se dize*; Concauidad entre viga y viga*; Conchauar*; Conciencia*; Condecender conel que algo pide*; Con sazon y tiempo*; Contemplar*; Contenerse o abstenerse*; Contiento*; Contraer parentesco*; Contricion tener: busca arrepentirse*; Conuiene a saber*; Coxcorron dar a otro con la mano*; Cruxir los dientes de 
frio*; Cuerço*; Cutaras o sandalias*; derecho*; Ceuil persona*; CircunCuzilla o tosca*; Çahareño. busca es- specto. busca auisado*; Cirial*; Çonquiuo*; Çanja para cimiento de pa- gotrear la vasija o cuero que no esta red*; Çarçaparrilla*; Cejo poner con- lleno*; Chirimia*; Chirriar las aues*; tra alguno*; Cenogil*; Certero que tira Chocarrear o dezir chufas. 